\title{
Substanz und Person
}

\section{Was ist eine Substanz?}

Das ist die erste Frage, die hier zu beantworten ist. Bei ihrer Beantwortung geht es um nichts anderes als um eine Klärung des Substanzbegriffs, an deren Ende eine Bedeutungsexplikation im carnapschen Sinn steht, ${ }^{1}$ also eine Definition des Wortes »Substanz«, die weder eine bloße Bedeutungsfestlegung noch eine bloße Bedeutungsanalyse ist, sondern Elemente von beiden enthält. Während eine Bedeutungsfestlegung mit einem neuen Wort einen neuen - oder auch alten - Begriff verbindet und eine Bedeutungsanalyse ein schon gegebenes Wort-BegriffVerhältnis nur betrachtet und diesbezüglich Behauptungen aufstellt, verbindet eine Bedeutungsexplikation mit einem alten Wort - wie es das Wort "Substanz" ja ist - einen neuen Begriff; welcher freilich, so lautet die Regel, erkennbar dem schon mit dem Wort verbundenen Begriff ähnlich sein soll bzw., wenn das Wort mehrdeutig ist, demjenigen Begriff ähnlich sein soll, auf den es einem ankommt, unter den mehreren Begriffen, die mit dem Wort verbunden werden. Während eine Bedeutungsfestlegung keiner Rechtfertigung bedarf und die Rechtfertigung einer Bedeutungsanalyse schlicht ihre evidente Wahrheit ist, liegt die Rechtfertigung einer Bedeutungsexplikation darin, dass sie für anstehende Zwecke der Klassifikation und Unterscheidung einen präzisen und nützlichen Begriff liefert. Man möchte mit ihm genaue und wichtige Unterscheidungen treffen; wenn möglich, sollte er bei der Theoriebildung eine zentrale Rolle spielen, was besagt: er sollte vernetzt mit vielen anderen Begriffen in vielen wahren generellen Aussagen seinen Auftritt haben.

Der Vorbereitung auf eine Bedeutungsexplikation dient ein eingehender Blick auf den Sprachgebrauch, in dem das Wort, das Gegenstand der Explikation ist, schon figuriert. Im Fall des Wortes »Substanz« ist das ein höchst heterogener Sprachgebrauch. Zunächst ist festzustellen, dass das Wort sowohl nichtrelational als auch relational verwendet wird. Die relationale Verwendung liegt vor, wenn man von »der Substanz von $x$ « oder, unter Verwendung des Genitivs, von »der Substanz des/der $x$ « spricht. Man meint damit dasselbe wie mit »der Seinskern von $x \ll$. Mit der Substanz von etwas mag eine Substanz im nichtrelationalen Sinn gemeint sein ich, z. B., würde nicht zögern zu sagen: meine Substanz - die Substanz von mir ist eine Substanz. ${ }^{2}$ Aber mit der Substanz von etwas kann auch eine Eigenschaft gemeint sein (etwa wenn man sagt, dass Vernünftigkeit die Substanz des Menschen

1 Vgl. Carnap 1956, 7-8; Carnap 1962, 3-8.

2 Wenn ich so spreche, spreche ich von der individualen Substanz von mir (und zwar von mir als Mensch). Siehe dazu des Weiteren die Fußnoten 6 und 20. 
sei), und eine Eigenschaft ist nun sicherlich keine Substanz im nichtrelationalen Sinn. Im Folgenden lasse ich die relationale Verwendung des Wortes »Substanz« (weitgehend) beiseite. Der Begriff, auf den es mir ankommt (und uns ankommen soll), ist im Gebiet der nichtrelationalen Verwendung des Wortes zu suchen.

Allerdings gibt es eine nichtrelationale Verwendung des Wortes "Substanz «, die für philosophische Zwecke irrelevant ist. Mein Schwager, der Pharmazeut ist, kündigt mir gelegentlich im Scherz an, dass er demnächst ein Pendant zu meinem Buch Ereignis und Substanz verfassen wird - nämlich ein Buch mit dem Titel Substanz und Ereignis. Das Wort »Substanz« hat eben auch einen chemisch-pharmazeutischen, nichtrelationalen Gebrauch. Wir wissen alle, mit welchem Ereignis bald zu rechnen ist, wenn uns in einem Roman erzählt wird, dass eine finstere Gestalt eine weiße Substanz heimlich in ein Weinglas schüttet (welche sich in dem darin befindlichen Wein spurlos auflöst).

Im Folgenden lasse ich die chemisch-pharmazeutische Verwendung des Wortes »Substanz« beiseite. Der Begriff, auf den es mir ankommt, ist klarerweise im Gebiet der philosophischen nichtrelationalen Verwendung des Wortes zu suchen. Damit steht der Explikator aber nun vor einem Berg ungeheurer Höhe und Tiefe - ein Sprachberg, der sich in den vergangenen zweieinhalbtausend Jahren allmählich aufgetürmt hat. Ich werde ihn nicht erkunden, nicht durchtunneln, nicht überqueren. Ignorieren kann ich ihn aber auch nicht. Ich weise also hin auf einige Aspekte des historisch gewachsenen philosophischen Gebrauchs des Wortes "Substanz« (oder seiner Äquivalente in anderen Sprachen) - Aspekte, die mir für die intendierte Bedeutungsexplikation wesentlich (ich könnte auch sagen: substantiell) erscheinen. Dementsprechend verwende ich diese Aspekte (explizit oder implizit), um zu einer Explikation der philosophischen Bedeutung von »Substanz« zu gelangen.

Dass eine Substanz etwas ist, von dem etwas ausgesagt werden kann, trägt nichts dazu bei, Substanzen zu charakterisieren; denn alles - jede Entität - ist etwas, von dem etwas ausgesagt werden kann: alles ist ein Prädikationssubjekt. Dass eine Substanz auch etwas ist, was selbst von nichts ausgesagt werden kann, leistet etwas mehr für die Charakterisierung von Substanzen, aber auch nicht viel: Ereignisse, Sachverhalte, ja selbst gewisse Universalien (nämlich Typenobjekte, ${ }^{3}$ wie z. B. der Kammerton A, oder die Oper Carmen) sind allesamt Entitäten, die von nichts ausgesagt werden können - sie sind aber keine Substanzen. Die Charakterisierung von Substanzen als Individuen unterscheidet sie von Sachverhalten und Typenobjekten, sowie von Begriffsobjekten (oder »Individualbegriffen«) - z. B. der Sinn (nicht der Bezug) der Kennzeichnung »der Präsident der USA im Jahre 2olo» - und von Propositionen - z. B. der Sinn des Satzes »Barack Obama ist Präsident der USA im Jahre

3 Typenobjekte sind Universalien, da sie der Exemplifikation (gewöhnlich der mehrfachen) fähig sind. $\mathrm{Zu}$ Typenobjekten und den anderen ontologischen Kategorien, von denen in diesem und dem nächsten Absatz die Rede ist, siehe Meixner 2004a. 
2010« - und übrigens auch von Meinong'schen Objekten (nur für den Fall, dass einer meint, manche Entitäten - als Entitäten angesehene Romangestalten etwa wären solche). Alle Substanzen sind Individuen, während die genannten Entitäten anderer Sorten zwar wie die Substanzen Objekte sind (d. h.: "gesättigte « Entitäten, Entitäten, die von nichts ausgesagt werden können), aber eben keine Individuen.

Jedoch ist die Charakterisierung von Substanzen als Individuen keineswegs eine hinreichende oder auch nur annähernd hinreichende: Es fehlt viel daran, dass jedes Individuum eine Substanz wäre. Alle Ereignisse sind Individuen (und folglich Objekte); aber kein Ereignis ist eine Substanz (wie gesagt). Und alle partikularisierten Eigenschaften - auch "Tropen «, "Momente», »abstrakte Teile«, »individualisierte Formen «, »individuelle Akzidenzien « genannt - sind Individuen, aber keine dieser Entitäten ist eine Substanz. Partikularisierte Eigenschaften sind unselbständige Individuen; Ereignisse sind Individuen mit intrinsischer zeitlicher Lokalisierung. Substanzen dagegen sind zwar Individuen, aber selbständige und solche ohne intrinsische zeitliche Lokalisierung. Ich halte fest:

SUB 1 Jede Substanz ist ein selbständiges Individuum ohne intrinsische zeitliche Lokalisierung.

Hiermit ist man nun erstmals in der Nähe eines präzisen und nützlichen Substanzbegriffs. Damit aus SUB1 eine Bedeutungsexplikation des Wortes "Substanz « wird, müsste man neben SUBı auch seine Umkehrung annehmen,

SUB2 Jedes selbständige Individuum ohne intrinsische zeitliche Lokalisierung ist eine Substanz,

und beide Aussagen - per fiat, aber durch ein wohlmotiviertes, keineswegs völlig freies, willkürliches fiat - zu Bedeutungswahrheiten erklären. Es ist das Besondere an SUB1, dass diese Schritte bei SUBı gerade eben rational möglich, wenn auch alles andere als rational zwingend erscheinen. Der Bedeutungsexplikator steht vor der Entscheidung, ob er für die wahrheitsgemäße Anwendbarkeit des Wortes »Substanz« semantisch noch mehr verlangen soll als das, was in SUBı hinter »ist « zum Ausdruck kommt, oder ob er das, was in SUBı hinter wist " zum Ausdruck kommt, für die wahrheitsgemäße Anwendbarkeit jenes Worte schon als bedeutungsmäßig notwendig und hinreichend ansetzen soll. Ich entscheide mich für das Letztere (und damit für einen weiten, ja minimalen Substanzbegriff):

SUBEXP $»$ Substanz $«=$ DefExp $"$ selbständiges Individuum ohne intrinsische zeit liche Lokalisierung ${ }^{4}{ }^{4}$

4 Lies: "Substanz « bedeutet per definitionem im Sinne einer Bedeutungsexplikation dasselbe wie »selbständiges Individuum ohne intrinsische zeitliche Lokalisierung “. 


\section{Individuum, selbständig und ohne intrinsische zeitliche}

\section{Lokalisierung}

Der Wert der Bedeutungsexplikation SUBEXP lässt sich steigern, indem man so weit wie möglich klärt, was mit dem Ausdruck »selbständiges Individuum « gemeint ist, und was mit dem Ausdruck »ohne intrinsische zeitliche Lokalisierung «.

Das Wort »Individuum«, für sich genommen, drückt einen ontologischen Kategorialbegriff aus, den man durch Anwendungsbeispiele - solche, wo er zutrifft, und solche, wo er nicht zutrifft - zwar erläutern kann, den man aber kaum bedeutungsexplikativ definieren kann - es sei denn durch eine Negativdefinition von etwas zweifelhaftem Wert:

»Individuum « = DefExp Objekt (d. h.: gesättigte Entität, Entität, die von nichts ausgesagt werden kann), das weder ein Sachverhalt, noch eine Proposition, noch ein Begriffsobjekt, noch ein Typenobjekt [noch ein Meinong'sches Objekt] ist $\ll$.

In einem gewissen Sinn gilt nicht nur für Individuen - also für Instanzen der zutreffenden Anwendung des Individuumbegriffs -, sondern auch für den Individuumbegriff selbst der alte ontologische Spruch: individuum est ineffabile. Daran ändert sich natürlich nicht das Geringste, wenn man statt von »Individuen« von »Partikularien « (Englisch: "particulars«) spricht. ${ }^{5}$

Was aber ist gemeint, wenn man sagt, dass etwas ein selbständiges Individuum ist? Diese Frage zu beantworten, ist alles andere als leicht. Die Schwierigkeit dabei ist diese: zu vermeiden, dass am Ende das Prädikat »selbständiges Individuum « auf rein gar nichts zutrifft oder bestenfalls nur auf sehr wenige Individuen, eventuell nur auf ein einziges. Definiert man z. B.: »Ein selbständiges Individuum ist ein Individuum, das (existiert und) zu seiner Existenz der Existenz von nichts anderem bedarf«, so ist schnell zu sehen, dass es keine selbständigen Individuen im Sinne dieser Definition gibt. Nichts - also insbesondere auch kein Individuum - kann existieren, ohne dass der Sachverhalt, dass es existiert, besteht. Jedes Individuum bedarf also zu seiner Existenz der Existenz von etwas anderem; kein Individuum ist also ein selbständiges Individuum.

Das muss nicht so sein, wenn man das Definiens der eben gegebenen Definition abschwächt: »Ein selbständiges Individuum ist ein Individuum, das zu seiner Existenz der Existenz von keinem anderen Individuum bedarf." Aber gemäß dieser Definition ist immer noch jedes Individuum kein selbständiges Individuum - also wegen SUBEXP auch keine Substanz -, dessen individuale Existenz (eine individualisierte Form) oder dessen individuale Natur (eine individualisierte Wesensform)

${ }^{5}$ Die Tatsache, dass »Individuum « wörtlich genommen dasselbe besagt wie »Atom «, ist im ontologischen Sprachgebrauch nicht präsent und darf unberücksichtigt bleiben. 
von ihm selbst verschieden ist. ${ }^{6}$ Ist nämlich die individuale Natur eines Individuums oder dessen individuale Existenz von ihm selbst verschieden, dann bedarf es zu seiner Existenz der Existenz eines anderen Individuums; denn es kann nicht existieren, ohne dass seine individuale Natur und seine individuale Existenz existieren, und diese sind nun einmal Individuen. Beispielsweise kann ich (ein gewisses Individuum) nicht existieren, ohne dass meine individuale Natur, mein individualisiertes Menschsein (ein gewisses anderes Individuum), existiert. Ich bin also gemäß der nun zur Debatte stehenden Selbständigkeitsdefinition für Individuen kein selbständiges Individuum, und folglich bin ich (gemäß SUBEXP) keine Substanz. Ja, es ist sehr zu vermuten, dass nicht eben viele Individuen gemäß jener Definition selbständige Individuen sind. Es ist in diesem Zusammenhang erwähnenswert, dass Thomas von Aquin im Effekt lehrt, dass das einzige Individuum, dessen individuale Existenz und dessen individuale Natur mit ihm selbst identisch ist, Gott ist. ${ }^{7}$ Daraus würde gemäß der in Betracht gezogenen Definition folgen, dass höchstens Gott ein selbständiges Individuum ist, und folglich gemäß SUBEXP, dass höchstens Gott eine Substanz ist.

Die Lehre von Gott als der einen Substanz wurde bekanntlich von Spinoza, aber nicht von Thomas von Aquin, vertreten. Jedoch, auch wenn Gott als Substanz zu betrachten ist, wofür ich plädieren würde (was gewissermaßen, freilich nur gewissermaßen, entgegen Thomas ist), ${ }^{8}$ so sollte der Substanzbegriff (entgegen Spinoza) nicht derart exklusiv sein, dass nur Gott eine Substanz ist. Eine weitergehende Liberalisierung der Definition von "selbständiges Individuum « ist demnach angesagt. Wie auch immer diese Liberalisierung ausfällt, das Ergebnis muss in Anbetracht des Ziels, einen hinreichend weiten Sinn mit dem Ausdruck mselbständiges Individuum « zu verbinden, um hinreichend vieles als Substanz gelten lassen zu können,

6 Beispielsweise gilt für jeden Menschen $x$ : (1.) die individuale Existenz von $x$ ist verschieden von $\boldsymbol{x}$, (2.) die individuale Natur von $\boldsymbol{x}$ (= die individualisierte Wesensform von $\boldsymbol{x}=$ das individualisierte Menschsein von $x$ ) ist verschieden von $x$. Die individuale Substanz eines Menschen (sein individualer Seinskern, seine Seele) darf nicht mit seiner individualen Natur (seinem individualen Wesen) gleichgesetzt werden. Dennoch gilt für jeden Menschen $x \operatorname{sehr}$ wohl auch dies: die individuale Substanz von $x$ ist verschieden von $x$.

7 Eine umfassende Rekonstruktion der in Thomas von Aquins Ontologie der Zusammensetzung (wozu auch seine Lehre von der Einfachheit Gottes gehört: siehe Summa Theologiae, I, qu. 3) verwendeten Begriffe und ihres Zusammentretens in Aussagen (samt Formalisierung) findet sich in Meixner 2016.

8 In S. Th. I, 3, 5 ("Utrum Deus sit in genere aliquo») argumentiert Thomas (sinngemäß), dass Gott wegen Seiner ontologischen Exzellenz in keiner Gattung ist, also auch nicht in der Gattung Substanz ist, also keine Substanz ist. Aber Thomas war in seiner Ablehnung der Substantialität Gottes keineswegs konsequent. Anderswo spricht er von der »divina substantia«, wieder anderswo, offenbar die Verlegenheit sehend, in der er steckt, bedient er sich des probaten Mittels der pseudo-dionysischen Superprädikation (eine Art von logischem "Wasch mir den Pelz, aber mach mich nicht nass ) und spricht von Gott als der »substantia supersubstantialis». Siehe Schütz 1958,781 f. 
das folgende Schema erfüllen: »selbständiges Individuum « bedeutet dasselbe wie »Individuum mit dem und dem Mindestgrad $y$ an Selbständigkeit«, wobei $y$ auf einer Skala zwischen o und 1 nicht unerheblich unter 1 liegt.

Benjamin Schnieder, der sich ausführlich mit dem Problem der adäquaten Bestimmung von Selbständigkeit (er spricht von Unabhängigkeit) bei der Substanzdefinition befasst hat, ${ }^{9}$ verfehlt das erwähnte Ziel bei weitem. Er meint, "[d]a Substanzen $[\ldots]$ von nichts permanent explanatorisch abhängig zu sein scheinen, verheißt der Begriff explanatorischer Abhängigkeit Gutes für Aristoteles' Idee [die Idee, Substanzen als ontologisch unabhängig anzusehen]; auf seiner Basis hat sie einen guten Stand « (Schnieder 2005, 77-78; zu »Aristoteles' Idee «: 55). Keineswegs. Gemäß Schnieder ist $x$ von nichts permanent explanatorisch abhängig genau dann, wenn für kein $y$ gilt, dass es ein F gibt, so dass gilt: $x$ existiert zu allen Zeitpunkten, zu denen es existiert, weil $y$ zu diesen Zeitpunkten $F$ ist (ebd., 77); wobei das »weil« begrifflich explanatorisch gemeint sein soll (ebd., 77, Fußnote; Näheres dazu in Schnieder 2004, 345-348). Wenn die explanatorische Unabhängigkeit von allem eine notwendige Bedingung des Substanzseins sein soll (wie es Schnieder offenbar haben möchte; in diesem Punkt wesentlich deutlicher ist seine finale Substanzdefinition in Schnieder 2004, 348), dann ist kaum etwas eine Substanz. Menschen jedenfalls sind demzufolge keine Substanzen, da ein Mensch zu allen Zeitpunkten, an denen er existiert, deshalb existiert, weil sein Körper zu diesen Zeitpunkten lebendig ist. ${ }^{10}$ Menschen und andere Lebewesen sind nun einmal permanent (begrifflich) explanatorisch abhängig von ihrem Körper.

Aber ich verlasse diese Baustelle nun (werde freilich zu ihr zurückkehren müssen) und frage vielmehr: Was meint im Definiens von SUBEXP der Ausdruck »ohne intrinsische zeitliche Lokalisierung«? Eine Entität hat eine intrinsische zeitliche Lokalisierung, wenn zu ihr selbst - als eine ontologische Konstituente von ihr - ein Ort in der Zeit gehört, d. h.: ein bestimmter Zeitpunkt oder eine bestimmte Menge von Zeitpunkten, typischerweise ein Zeitintervall. Paradigmatische Beispiele für Entitäten mit intrinsischer zeitlicher Lokalisierung sind Ereignisse: Zu jedem Ereignis gehört selbstinnerlich sein Ort in der Zeit; es wäre nicht dieses Ereignis, hätte es einen anderen zeitlichen Ort. Es hat diesen zeitlichen Ort auch dann, wenn es nicht geschieht; aber geschieht es, wird es wirklich, dann füllt es neben anderen wirklichen Ereignissen, die intrinsisch ebenfalls diesen zeitlichen Ort haben, genau diesen Ort aus. Ist der dem Ereignis intrinsische zeitliche Ort mehr als ein Zeitpunkt (und nur dann), so hat das Ereignis in sich echte zeitliche Teile, Phasen, wie man auch sagt. Ist der dem Ereignis intrinsische zeitliche Ort ein Zeitintervall (und nur dann), so hat das Ereignis in sich eine gewisse positive zeitliche Ausdehnung, eine echte Dauer, wie man auch sagt.

\footnotetext{
${ }^{9}$ Vgl. Schnieder 2004, Kap. 3-6, sowie Schnieder 2005.

${ }^{10}$ Die Biologie definiert, was es heißt, lebendig zu sein, welche Beschaffenheit eines Körpers das ist.
} 
Substanzen aber haben - wie auch Entitäten, die nicht der Kategorie der Individuen angehören (beispielsweise zeitlich unspezifische Universalien oder zeitlich unspezifische Sachverhalte) - keine intrinsische zeitliche Lokalisierung. Daraus folgt ipso facto, dass Substanzen in sich auch keine zeitlichen Teile und keine Dauer - keine zeitliche Ausdehnung - haben. In sekundärer, analogischer Sprechweise können einer Substanz allerdings eine Dauer und sogar zeitliche Teile zugesprochen werden, sofern es nur ein wirkliches Ereignis gibt, mit welchem sich die Substanz in der Zeit manifestiert. Als die Dauer der Substanz gilt (analogischsekundär) die Dauer jenes Ereignisses, als die zeitlichen Teile der Substanz gelten die zeitlichen Teile jenes Ereignisses. Bei uns Menschen etwa ist das fragliche Manifestationsereignis einfach unser jeweiliges (wirkliches) Leben. Es ist hier wichtig festzuhalten: "ohne intrinsische zeitliche Lokalisierung « heißt nicht »ohne Bezug zur Zeit«, heißt nicht einmal »ohne engen Bezug zur Zeit«. Die Beziehung einer Substanz zur Zeit ist vielmehr gerade so eng, wie ihre Beziehung zu ihrem Manifestationsereignis ist. Da es für jede Substanz essenziell ist, welche - so und so inhaltlich beschaffenen, so und so strukturierten - zueinander alternativen möglichen Ereignisse als ihr Manifestationsereignis in Frage kommen (gewiss sind das nicht alle möglichen Ereignisse), ist das Verhältnis zwischen einer Substanz und ihrem Manifestationsereignis gewiss kein rein äußerliches. Freilich ist dieses Verhältnis, wie es auch ausfällt, ein kontingentes: es hätte nun eben auch anders ausfallen können - wie es übrigens auch kontingent sein mag, dass eine Substanz überhaupt ein Manifestationsereignis hat. Letzteres wäre unbedingt dann so, wenn jede Substanz auch ohne ein Manifestationsereignis als Substanz existieren kann. Nur wenn eine Substanz kein Manifestationsereignis hat - was aber vermutlich bei keiner einzigen (existierenden) Substanz ${ }^{11}$ tatsächlich der Fall ist -, kann man sagen, sie sei »außerhalb der Zeit« oder gar »überzeitlich «.

Es ist hier auch der Ort, dem Klischee von der Unveränderlichkeit - oder wie man sich gerne ausdrückt: Starrheit - der Substanz entgegenzutreten. Selbstverständlich sind Substanzen weder unveränderlich noch starr: sie haben zu verschiedenen Zeiten verschiedene Eigenschaften - welche Eigenschaften das sind, lässt sich an ihren jeweiligen Manifestationsereignissen ablesen. ${ }^{12}$ Es ist versucht worden, sogenannten Enduranten den ontologischen Garaus zu machen, und damit auch Substanzen - denn wegen des Merkmals, ohne intrinsische zeitliche Lokalisierung zu sein, sind Substanzen Enduranten. Angeblich seien Enduranten -

11 Die Existenz liegt bereits im Begriff der Substanz, wenn sie von der Selbständigkeit (die gemäß SUBEXP in die Definition von »Substanz« eingeht) beinhaltet wird. Und wie sollte Selbständigkeit Existenz nicht (analytisch) beinhalten? Etwas, was nicht existiert, wird man kaum als "selbständig« bezeichnen.

12 Eine unveränderliche Substanz, die ein Manifestationsereignis hat, müsste ein monotones, d. h., ein zu allen seinen Zeiten stets denselben Momentanzustand präsentierendes Manifestationsereignis haben. Doch nicht einmal ein tief im Inneren eines Berges ruhender Diamant hat ein solches Manifestationsereignis. 
inakzeptablerweise - der intrinsischen Veränderung, etwa des Gestaltwechsels, unfähig. ${ }^{13}$ Angeblich liege in dem Gedanken, dass numerisch ein und dasselbe Etwas einmal die Eigenschaft $F$ habe, ein andermal aber nicht, überhaupt ein Widerspruch (so Erwin Tegtmeier in Tegtmeier 1992, 76, und so auch, im Effekt, schon David Hume) ${ }^{14}$ Doch der einzige Preis, den die Veränderung, und auch die intrinsische Veränderung, von Enduranten und folglich von Substanzen wirklich fordert, ist die Zeitabhängigkeit des Habens von Eigenschaften (m. a. W.: der Eigenschaftsexemplifikation), und das ist wahrlich kein Grund, Enduranten zu verwerfen.

\section{Arten von Substanzen}

Mit der Annahme von SUBEXP entscheidet man sich für einen minimalen Substanzbegriff. Man hätte den Substanzbegriff auch stärker aufladen können. Dafür gibt es philosophiehistorische Motive. Als den ersten Satz seines Spätwerks Principes de la nature et de la grâce fondés en raison, aus dem Jahre 1714, setzt Leibniz: »La Substance est un Être capable d'Action ${ }^{15}$ - „Die Substanz ist ein Seiendes, das der Handlung fähig ist.« Dementsprechend hätte man die Fähigkeit, kausal wirksam zu sein, oder auch gleich das kausale Wirksamsein selbst in den Begriff der Substanz aufnehmen können (denn was ist eine Fähigkeit ohne ihre Realisierung?), das Definiens von SUBEXP entsprechend verstärken können. Ich ziehe es vor, innerhalb des Genus Substanz eine Spezies zu unterscheiden: aktive Substanz - Substanz, die kausal wirksam ist. Gemäß SUBEXP ist klar, dass eine Substanz weder ein Ereignis, noch ein Sachverhalt, noch eine Eigenschaft ist. Ereignis, Sachverhalt, Eigenschaft das sind die gängigen Kategorien (in der Reihenfolge der Häufigkeit ihrer Nennung) von Entitäten, die als kausal wirksam (m. a. W.: als Ursachen) angesehen werden. Die Kausalität einer Substanz muss eine andere sein als die Kausalität von Ereignissen, Sachverhalten, Eigenschaften. Man spricht von Agenskausalität oder Akteurskausalität - deren Explikation und Verteidigung allerdings ein Kapitel für sich ist. Die paradigmatischen Agensursachen - nämlich wir selbst - sind aber jedenfalls Substanzen (wie das folgt, dass wir Substanzen sind, dazu siehe weiter

13 Siehe David Lewis 1986, 203-204. Ich kritisiere Lewis' Argumentation eingehend in Meixner 2006, 94-99.

14 Siehe dazu des Näheren: Meixner 2002, 442, 448-449. Die korrekte Formulierung des sogenannten Leibnizprinzips der Identität mit zeitbezogener Eigenschaftshabe lautet: Wenn $a$ und $b$ identisch sind, dann haben zu $\ell a$ und $b$ dieselben Eigenschaften (für alle Zeitpunkte $t$ ). Die inkorrekte Formulierung lautet: Wenn $a$ und $b$ identisch sind, dann haben zu $t$ und zu $t^{\prime} a$ und $b$ dieselben Eigenschaften (für alle Zeitpunkte $t$ und $t^{\prime}$ ). Sie ist inkorrekt, weil sie in die Identität gleich auch noch die Unveränderlichkeit einbaut: Wegen $a=a$ folgt ja mit ihr: $a$ hat zu $t$ und zu $t^{\prime}$ dieselben Eigenschaften (für alle Zeitpunkte $t$ und $t^{\prime}$ ).

15 Leibniz 1985,414 
unten), und man kann mit Recht fragen, ob außerhalb der Kategorie der Substanz für Agensursachen noch Platz ist. ${ }^{16}$

Kausalität ist das eine, was mit Substanzen in bedeutsamer Weise in Verbindung gebracht werden kann (ohne dass man es doch in den Substanzbegriff selbst hineinnehmen sollte); Bewusstsein ist das andere. Innerhalb des Genus Substanz ist folglich eine weitere Spezies zu unterscheiden: bewusste Substanz - Substanz, die ein Bewusstseinssubjekt ist. Hiernach ist eine bewusste Substanz aus trivialen begrifflichen Gründen ein Bewusstseinssubjekt. Die interessante Frage ist, ob ein Bewusstseinssubjekt immer eine Substanz ist. Die Tendenz der neuzeitlichen Philosophie ist - nach anfänglicher deutlicher Bejahung dieser Frage bei Descartes insgesamt ein immer ausdrücklicheres Abrücken von der Bejahung. Das Problem für den Substanzstatus (im Sinne von SUBEXP) von Bewusstseinssubjekten ist nicht das Individuumsein oder das Ohne-intrinsische-zeitliche-Lokalisierung-sein (die Phänomenologie des Bewusstseins - wie sie insbesondere Edmund Husserl erarbeitet hat - lässt bei dem, der auf introspektive Evidenz überhaupt etwas gibt, keinen Zweifel daran, ${ }^{17}$ dass Bewusstseinssubjekte Individuen ohne intrinsische zeitliche Lokalisierung sind); das Problem für den Substanzstatus von Bewusstseinssubjekten ist das Selbständigsein. Sind sie selbständige Individuen? Ob sie dies sind oder nicht, dafür (beides $\mathrm{Mal}$ ) kommt es offensichtlich darauf an, was man unter »selbständiges Individuum “ versteht. Einerseits lässt sich im Sinne Descartes' dafür argumentieren, dass Bewusstseinssubjekte Individuen sind, die zu ihrer Existenz. der Existenz von keinem physischen Individuum metaphysisch bedürfen. ${ }^{18} A n$ dererseits legt die Hirnforschung mehr als nur nahe, dass Bewusstseinssubjekte Individuen sind, die zu ihrer Existenz der Existenz von ganz bestimmten physischen Individuen naturgesetzlich bedürfen. Haben Bewusstseinssubjekte also nun einen hinreichenden Grad an Selbständigkeit dafür, sie als »selbständige Individuen“ bezeichnen zu dürfen? (Vgl. die diesbezüglichen Ausführungen im vorausgehenden Abschnitt.) Mein Vorschlag wäre, die Frage mit »ja« oder »nein« zu beantworten je nachdem, ob ein Bewusstseinssubjekt aktiv - m. a. W.: kausal wirksam - ist oder nicht. Ist ein Bewusstseinssubjekt kausal wirksam, aktiv, dann ist es - gilt es als - ein selbständiges Individuum ${ }^{19}$ und ist also (da alles übrige Erforderliche gegeben ist) eine Substanz. Ist ein Bewusstseinssubjekt dagegen nicht kausal wirk-

${ }^{16}$ Substanzen und ihrer Kausalität gehe ich ausführlich nach in Meixner 1997. Dort wird auch die Kausalität von Aggregationen von Substanzen betrachtet.

17 Man beachte: Bewusstsein ist dasjenige Sein, dessen erscheinendes Sein (phänomenales Sein mit seinem So-sein identisch ist. (Physisches Sein dagegen ist gerade kein Sein, dessen erscheinendes Sein mit seinem So-sein identisch ist.)

18 Vgl. dazu: Meixner zoo4b, Kap. III.

19 Zur Stärkung dieses Vorschlags sei gesagt, dass kausal wirksame Bewusstseinssubjekte Agensursachen sind - und alle Agensursachen sind Erstursachen (unverursachte Ursachen!da eine Wirkung - ein Verursachtes - stets ein Ereignis oder Sachverhalt ist, eine Agensursache aber in keinem Fall ein Ereignis oder Sachverhalt ist. 
sam, nicht aktiv, dann ist es kein selbständiges Individuum und also auch keine Substanz. Sollte es sich also im Widerspruch dazu, wie wir uns selbst erscheinen und selbst verstehen, herausstellen, dass (reale) menschliche Bewusstseinssubjekte bloße Epiphänomene sind, ohne jede kausale Wirksamkeit, dann stünde damit fest, dass menschliche Bewusstseinssubjekte keine Substanzen sind. Einstweilen jedoch besteht noch kein echter Anlass dazu, davon abzugehen, dass (reale) menschliche Bewusstseinssubjekte - wir also - eine kausale Wirksamkeit haben. Damit ergibt sich (auf der Grundlage des schon Gesagten), dass wir Substanzen sind, und zwar aktive und bewusste Substanzen:

ARG Wir sind menschliche Bewusstseinssubjekte, ${ }^{20}$ also: Individuen ohne intrinsische zeitliche Lokalisierung (wie unser Selbsterscheinen zeigt); wir sind zudem kausal wirksam (gemäß unserem Selbsterscheinen und Selbstverstehen), also: selbständige Individuen (gemäß der gerade vorgetragenen Argumentation). Folglich sind wir Substanzen (gemäß SUBEXP), und zwar aktive (da kausal wirksam) und bewusste (da Bewusstseinssubjekte).

Aktive Substanz und bewusste Substanz sind also zwei sich extensional überschneidende Spezies von Substanz, und in Konjunktion bilden sie eine dritte Spezies von Substanz: aktive und bewusste Substanz. Eine vierte Spezies von Substanz ist die vernunfthabende Substanz. Nicht jede aktive und bewusste Substanz ist eine vernunfthabende. Doch jede vernunfthabende Substanz ist eine aktive und bewusste. Denn eine Substanz, die kein Bewusstseinssubjekt, nicht bewusst ist, kann man nicht als »vernunfthabend « bezeichnen: dazu, Vernunft zu haben (im eigentlichen, vollen Sinn), gehört per se, ein Bewusstseinssubjekt zu sein. Und eine Substanz, die nicht kausal wirksam, nicht aktiv ist, kann man ebenfalls nicht als "vernunfthabend « bezeichnen: dazu, Vernunft zu haben (im eigentlichen, vollen Sinn), gehört per se, ein Handlungssubjekt zu sein; aber ohne kausal wirksam zu sein, ist das Letztere nicht zu haben.

Die differentiae specificae Bewusstheit, Aktivität und Vernunfthabe sind hier im Sinne von Vermögen zu verstehen, die selbstverständlich nicht reine Vermögen sind, sondern auch tatsächlich aktual manifestiert werden. Nicht nötig ist es aber dafür, dass ein Individuum zu einem Zeitpunkt bewusst ist, aktiv ist, Vernunft hat,

${ }^{20}$ In näherer Analyse sind menschliche Bewusstseinssubjekte solche Bewusstseinssubjekte, die aus einem Körper menschlicher Natur naturgesetzlich emergieren und »zu ihm hin sind: intentional in vieler Hinsicht auf ihn intensiv bezogen sind. Ein menschliches Bewusstseinssubjekt ist offensichtlich kein Mensch (der ja, als solcher, ein so und so konfigurierter menschlicher - Körper ist, während ein menschliches Bewusstseinssubjekt kein solcher Körper ist, sondern nur einen solchen hat); aber zweifelsohne ist ein menschliches Bewusstseinssubjekt der individuale Seinskern eines Menschen: seine individuale Substanz ("Substanz« hier relational gebraucht). Mit dem Wort »ich « kann der ganze Mensch gemeint sein oder eben dessen individualer Seinskern. 
dass es das jeweils entsprechende Vermögen zum fraglichen Zeitpunkt aktual manifestiert. Mit anderen Worten: Wenn jemand tief schläft, aber in wenigen Stunden erwachen wird, so ist er, während er tief schläft, immer noch ein bewusstes, aktives und vernunfthabendes Individuum - obwohl die entsprechenden Vermögen in der fraglichen Zeit ohne Aktualmanifestation sind.

Es sei hinzugefügt, dass die beschriebenen Spezies von Substanz sehr wohl durchlässig sind. Ein natürlicher Aufstiegsweg ist dieser: Ein Individuum, das ohne intrinsische zeitliche Lokalisierung ist, gelangt zu rudimentärem Bewusstsein (und damit allein schon zur Existenz) und gleichzeitig zu rudimentärer kausaler Wirksamkeit. Es wird damit zur rudimentären Substanz. Im Einklang mit der Entwicklung seines Körpers wird dann das Individuum zur entwickelten Substanz. Das geschieht natürlicherweise (und bei allen Mitgliedern einer biologischen Spezies in annähernd gleicher Weise) durch die Steigerung der eigenen kausalen Wirksamkeit, die natürlicherweise (und zwar im Sinne des evolutionsbiologischen Nutzens) verbunden ist mit der Steigerung der eigenen Bewusstheit. Darüber hinaus wird ein Individuum, das einem menschlichen Körper einwohnt, wenn alles gut geht, wenig später zur vernunfthabenden Substanz, in einer ersten Vollendung seiner Bewusstheit und Aktivität. Die Vernunfthabe wiederum lässt Grade zu und kann durch ausbildende Förderung außerordentlich gesteigert werden.

Leider wird ein solcher natürlicher Aufstiegsweg, sofern das fragliche Individuum lange genug existiert, irgendwann durch einen ebenso natürlichen Abstiegsweg komplementiert (wie wir alle wissen). Die Eigenschaften der Bewusstheit, Aktivität, Vernunft und der unmittelbare Träger dieser Eigenschaften gehen hervor aus der physischen Organisation eines geeigneten Nervensystems und werden von ihr gestützt (werden von ihm aber weder konstituiert noch im eigentlichen Sinne verursacht, ganz wie die von einem Körper ausgehende Schwerkraft von diesem weder konstituiert noch im eigentlichen Sinn verursacht wird); mit dem Verfall jenes Nervensystems verfallen auch sie - so der natürliche Weg.

Im Sinne der klassischen Person-Definition des Boethius - »rationabilis naturae individua substantia - lassen sich vernunfthabende Substanzen auch als personale Substanzen bezeichnen (und umgekehrt personale als vernunfthabende). Jede personale Substanz ist zweifellos eine Person. Ist aber auch jede Person eine personale Substanz? Oder äquivalent: Ist jede Person eine Substanz? Boethius hätte diese Frage bejaht; er definiert ja Personen als gewisse Substanzen. Aber natürlich ist die Frage mit dem bloßen Verweis auf die klassische Antwort in keiner Weise beantwortet.

Ich werde sie aber auch nicht beantworten, denn sie ist gewissermaßen müßig, da wir nicht nur aktive und bewusste Substanzen sind, sondern auch Vernunft haben - Selbstreflexionsvermögen, Begriffsfähigkeit, Freiheit -, also vernunfthabende Substanzen sind, also personale Substanzen sind und mithin Personen. Ob es nun Personen gibt oder geben kann, die keine Substanzen sind, ist angesichts dessen, dass wir selbst Personen sind, die Substanzen sind, zweitranging, jedenfalls vom 
rein anthropologischen Standpunkt aus, auf den ich mich hier beschränken will. Vom theologischen und vom allgemein-zoologischen Standpunkt aus ließen sich gegen die These, dass alle Personen Substanzen sind, Bedenken vortragen. Gibt es nicht nichtmenschliche Personen, die keine Substanzen sind, nämlich mindestens zwei der drei Personen der Trinität, oder auch - am anderen Ende des Spektrums gewisse Tierpersonen (der treue Hund, das verständige Pferd)? So eindeutig mit »a« zu beantworten, wie die Frage es suggeriert, ist die Frage aber nicht. Hält man sich in der Auffassung des Substanzbegriffs streng an SUBEXP und an die Erläuterungen der in SUBEXP figurierenden Begriffe, so hängt »Ja« oder »Nein « hier am Ende davon ab, ob die fraglichen Personen der Trinität oder die fraglichen Tierpersonen einen hinreichenden Grad an Selbständigkeit haben (was wiederum auf das Gegebensein oder Nichtgegebensein eigener kausaler Wirksamkeit bei ihnen hinauslaufen dürfte). Wenn $j a$, dann sind sie jedenfalls im Sinne von SUBEXP nun eben doch Substanzen.

\section{Blindheiten und Erhellungen}

$\mathrm{Zu}$ den philosophischen Klischees, die mit Substanzen verbunden werden, gehört auch deren angebliche Isoliertheit, neben ihrer angeblichen Starrheit. Die Tendenz der nachmittelalterlichen Philosophie geht - trotz Leibniz, trotz Spinoza dahin, Substanzen aus der Ontologie heraus zu drängen, sie zu etwas von der menschlichen Erkenntnis gänzlich Abgeschnittenem mit bloß postulierten Funktionen zu machen - Funktionen zudem, für die man, so stelle sich angeblich heraus, Substanzen gar nicht brauche. Auf das überflüssige Je-ne-sais-quoi »Substanz" (ist man mit ihr fertig, so ist - gefühlt - nur noch die Worthülse übrig) kann man gut verzichten - und muss man ja verzichten gemäß Ockhams Regel der ontologischen Sparsamkeit (und auf dem Gipfel der Rationalität wird man sich dann fühlen). Die Tendenz der neuzeitlichen Philosophie geht dahin, Substanzen zunächst erkenntnismäßig zu isolieren, sie für unerkennbar zu erklären; dann Substanzen auch funktionell zu isolieren, ihnen die ihnen (dennoch noch) zugeschriebenen Funktionen abzuschneiden. Den verstümmelten Torso schließlich wirft man auf den Misthaufen der Philosophiegeschichte. Es ist ein schwacher Trost, dass der Misthaufen sich früher oder später verwandelt in das Eldorado der Philosophiegeschichtsschreibung - verstanden als ein Teilgebiet der Archäologie.

Der neuzeitlichen Tendenz wider die Substanz liegt Substanzblindheit zugrunde, ja man könnte geradezu von einer Art Idiotie sprechen. Das ist nicht zu viel gesagt. David Hume hat sein gewohnter Scharfsinn verlassen, wenn er, ausgerechnet um zu leugnen, dass er zu verschiedenen Zeiten identisch gegeben ist, sich als zu verschiedenen Zeiten identisch gegeben voraussetzt (und zwar so gut wie ausdrücklich): 
For my part, when I enter most intimately into what I call myself, I always stumble on some particular perception or other [...] I never can catch myself at any time without a perception, and never can observe anything but the perception. [...] If any one [...] thinks he has a different notion of himself, I must confess I can reason no longer with him. [...] He may, perhaps, perceive something simple and continued, which he calls himself; though I am certain there is no such principle in me. But setting aside some metaphysicians of this kind, I may venture to affirm of the rest of mankind, that they are nothing but a bundle or collection of different perceptions, which succeed each other with an inconceivable rapidity, and are in a perpetual flux and movement. ${ }^{2 l}$

Kann das Ich, welches zu verschiedenen Zeiten - den Zeiten »when I enter most intimately into what I call myself «- urteilt, dass es zu diesen Zeiten nicht zu sehen ist, sondern dass nur vorübereilende Perzeptionen zu sehen sind, und welches wieder zu einer anderen Zeit (der der Niederschrift des Treatise) von diesen Urteilen berichtet - kann dieses Ich zu all jenen Zeiten wirklich nicht präsent gewesen sein, oder in jenen Perzeptionen aufgehen ("which succeed each other with an inconceivable rapidity ()? Wohl kaum. Dennoch halten auch heute noch sehr viele Philosophen - und gewiss die Mehrheit derjenigen analytischen Philosophen, die überhaupt Introspektion akzeptieren - Humes Selbstbericht für evidentermaßen im Wesentlichen korrekt. Von einer phänomenologischen, im vollen Sinne empirischen (hier: auto-empirischen) Evidenz der Korrektheit kann aber keine Rede sein. Vielmehr ist der gewöhnlich unausgesprochene, sehr dogmatische Gedankengang, welcher der so beliebten Übernahme einer humeschen Bewusstseinskonzeption zugrunde liegt, der folgende "Schluss«: Eine solche Bewusstseinskonzeption ist anti-cartesianisch; folglich muss sie - im Wesentlichen - korrekt sein. ${ }^{22}$

Wer sich eine humesche Bewusstseinskonzeption zu eigen macht, versperrt sich damit den Königsweg zur Substanz, der nun eben über das auto-empirisch adäquat gesehene Bewusstseinssubjekt führt (welches, ohne intrinsische zeitliche Lokalisierung, zu verschiedenen Zeiten vollständig präsent ist). Über das Bewusstseinssubjekt führt auch der Königsweg zu den Relationen, in die Substanzen eintreten. Anders gesagt: Über Substanzen und ihre Beziehungen (zu so vielem) belehrt am

21 Hume $1962,301-302$.

${ }^{22}$ Gemäß der humeschen Bewusstseinskonzeption verläuft ein Bewusstsein ohne ein zu allen Bewusstseinszeiten vollständig anwesendes (also durch jene Zeiten nicht innerlich bestimmtes) Bewusstseinssubjekt, ohne ein Bewusstseinssubjekt, das keine intrinsische zeitliche Lokalisierung hat. Passend hierzu erscheinen Personen nicht als Substanzen, sondern als Ereignisse. Prominente neuere Vertreter einer solchen Auffassung von Bewusstseinssubjekt und Person sind beispielsweise David Lewis und Derek Parfit (siehe dazu Meixner 2006, 99-105). Ein prominenter Vertreter der Auffassung im 19. Jahrhundert ist William James (siehe James 1950, Kap. X). Auch für Franz Brentano ist es mzunächst eine offene Frage, ob der Fortbestand des Ich das Beharren ein und desselben einheitlichen Dinges oder etwa eine Aufeinanderfolge verschiedener Dinge sei, von welchen nur das eine an das andere sich anschließt und sozusagen an seine Stelle tritt « (Brentano 1973, 239). 
meisten die adäquate Betrachtung des eigenen Ichs. Diese Betrachtung enthüllt eine bewusste und aktive (vgl. ARG) Substanz, sogar eine vernunfthabende (personale), die in vielen Weisen mit der Welt befasst ist. Diese Weisen spiegeln sich öfentlich (intersubjektiv) und stabil in den Aussageformen unserer Sprache wieder: »ich sehe (sah, werde sehen) ...«, »ich fühle (fühlte, werde fühlen) ...«, »ich meine (meinte, werde meinen) ...., »ich glaube (glaubte, werde glauben) ...«, »ich will (wollte, werde wollen) ...«, »ich kann (konnte, werde können) ...«, »ich denke (dachte, werde denken) ...«, wich tue (tat, werde tun) ...«, »ich rätsele (rätselte, werde rätseln) ...«, »ich sage (sagte, werde sagen) ...», »ich erlebe (erlebte, werde erleben) .... usw. usf. Das Präteritum und das Futur dieser Aussageformen der Ersten Person sind deshalb beigefügt, weil die menschliche personale Substanz (die mit »ich« sich selbst nennt) mit den Tempora ihre jeweils vollständige Präsenz zu getrennten Zeiten - und damit auch ihre Identität über die Zeit hinweg: ihre zeitbezogene Identität ohne intrinsische zeitliche Lokalisierung - ganz natürlich zum Ausdruck bringt, etwa so: »Ich fühlte eine Zeit lang einen Schmerz im Schulterbereich, den ich nun nicht mehr fühle. Ich tat manche Dinge nur mit Mühe, die ich jetzt wieder ohne Mühe tun kann. Manche Bewegungen konnte ich gar nicht ausführen. Ich rätselte, woher der Schmerz wohl kommen mochte, und glaubte, er komme von einem Muskelriss. Jetzt ist es mir gleichgültig, woher er kam. Aber hoffentlich werde ich diesen Schmerz, nicht wieder fühlen."

Mit diesem Anwendungsbeispiel ist nun auch schon deutlich, für was die Auslas-

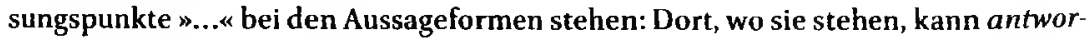
tend zum Ausdruck gebracht werden, wonach durch eine Frage nach dem Was des (vergangenen, gegenwärtigen, zukünftigen) Sehens, Fühlens, Meinens, Glaubens, Wollens, Könnens, Denkens, Tuns, Rätselns, Sagens, Erlebens usw. gefragt wird (nach dem Muster: "Ich erlebe.« - »Was erlebst du? - »Ich erlebe ... [das und das].«). Die genannten Weisen des Weltbezugs einer menschlichen personalen Substanz (und viele andere solche Weisen) sind Weisen der Intentionalität. In ihren Erfullungen ${ }^{23}$ sind sie stets auf etwas bezogen: man nennt es [ [ihr jeweiliges] intentionales Objekt ${ }^{24}$ (und dessen partikulare Beschreibung füllt die durch die

23. Weisen der Intentionalität sind Relationen, also mehrstellige Universalien. Eine Universalie ist erfullt, wenn (und nur wenn) ihre offenen Stellen durch dafür geeignete Entitäten gefüllt sind und das Ergebnis nicht nur ein Sachverhalt, sondern auch eine Tatsache ist. Eine ontologische Besonderheit von Universalien ist, dass sie nicht »wieder ausgeleert« werden müssen, um mehrfach gefüllt, und gegebenenfalls mehrfach erfüllt, zu sein. Wenn man das als paradox ansieht, hat man eine unpassende, nämlich physisch-konkrete Vorstellung von Universalien. Universalien sind keine Eimer und keine Steckreihen.

${ }^{24}$ Gewöhnlich sind intentionale Objekte keine Erlebnisse, aber bej den Bewusstseinsweisen des Fühlens und Erlebens sind auch ohne explizit reflexive Akte Erlebnisse als intentionale Objekte gegeben. Eine Schmerzepisode ist ein (stets unangenehmes, bisweilen unerträgliches) Erlebnis - und man fühlt und erlebt sie unmittelbar mit dem Schmerz selbst, also auch es (das Erlebnis, das sie ist). 
Auslassungspunkte »...« in den korrespondierenden Aussageformen bezeichnete Leerstelle).

Manche der Intentionalitätsweisen sind eher generisch, wie Tun, Fühlen und insbesondere Erleben (welches die unspezifischste Weise der Intentionalität in dem Sinne ist, dass jede Intentionalitätsweise F eine Weise des Erlebens ist, aber freilich ohne dass generell aus $» x$ Ft $y \ll » x$ erlebt $y \ll$ folgt); manche eher spezifisch, wie Rätseln und Sagen. Manche der Füllungen (zu diesem Begriff siehe Fußnote 23) von Intentionalitätsweisen sind nicht über das Bewusstsein hinausweisend, andere sind über das Bewusstsein hinausweisend. ${ }^{25}$ Das Unterscheidungskriterium zwischen nicht über das Bewusstsein hinausweisend und über das Bewusstsein hinausweisend ist - als konditionale Frage formuliert - dieses: Wenn es einem scheint, dass man in der Weise $F$ der Intentionalität auf das und das intentionale Objekt bezogen ist, ist es dann notwendig auch wirklich so - oder aber nicht? Wenn Ersteres die Antwort ist (»Es ist notwendig auch wirklich so «), dann ist die fragliche Füllung der Intentionalitätsweise $F$ (nämlich: dass man [jetzt] in der Weise $F$ auf das und das intentionale Objekt bezogen ist) nicht über das Bewusstsein hinausweisend; wenn Letzteres die Antwort ist, dann ist jene Füllung über das Bewusstsein hinausweisend.

Alle Intentionalitätsweisen sind experientiell, mit anderen Worten: sie sind Erlebnisweisen, was aber nicht bedeutet, dass sie alle rein passiv wären. Rein passiv sind manche Formen des Fühlens, oder die eine oder andere spezielle Form des Glaubens. Demgegenüber haben die meisten Intentionalitätsweisen eo ipso einen aktiv-kausalen Aspekt oder schließen einen solchen jedenfalls nicht eo ipso aus. Der aktiv-kausale Aspekt ist am augenfälligsten beim Tun (und seinen vielen, vielen Unterarten). ${ }^{26}$ Es mag merkwürdig erscheinen, dass Tun - als Intentionalitätsweise eine Erlebnisweise sein soll. Wenn ich aber sage »ich tue [das und das] «, so ist damit behauptet, dass die Intentionalitätsweise Tun in bestimmter Weise erfüllt ist, dass eine bestimmte Füllung von ihr eine Tatsache ist, und zu dieser (behaupteten) Erfüllung gehört, wenn sie existiert, unweigerlich und zentral das Vorliegen eines

25 Dass eine Füllung einer Intentionalitätsweise (durch eine Zeit, ein geeignetes Subjekt und ein geeignetes Objekt) über das Bewusstsein hinausweisend ist, bedeutet, dass die Bedingungen dafür, ob sie auch eine Erfüllung jener Intentionalitätsweise ist, partiell außerhalb des Bewusstseins liegen (d.h.: außerhalb des Bewusstseins desjenigen Bewusstseinssubjekts, dem es im Sinne der Füllung gegebenenfalls so schiene, als stünde es in der fraglichen Intentionalitätsweise zu einem gewissen intentionalen Objekt).

${ }^{26}$ Tun zerfällt zunächst in körperliches Tun und geistiges Tun. Arten des körperlichen Tuns sind z. B. alle Handlungsweisen mit der Hand (gewöhnlich unter Beteiligung des Arms): Zeigen, Antippen, Wegwischen, Winken, Festhalten, Loslassen, Nehmen, Geben, Schlagen, usw. Arten des geistigen Tuns sind z. B. alle Formen des urteilsbezogenen Denkens: im Denken In-Erwägung. ziehen, Abwägen, Ausschließen, Beiseitelassen, Verneinen, Bejahen, Begründen, Erklären usw. (Die genannten Beispiele legen nahe: Geistiges Tun äußert sich in körperlichem - insbesondere verlautendem - Tun; körperliches Tun ist geistig durchdrungen.) 
Erlebnisses des Tuns (bei mir). Nicht anders (sondern genauso, freilich mutatis mutandis) verhält es sich, wenn ich sage, »er/sie tut [das und das] «.

Diese Ausführungen mögen genügen, eine Vorstellung von der Reichhaltigkeit und Komplexität des intentionalen (meistens kausal-intentionalen) Weltbezugs menschlicher personaler Substanzen zu geben. Welche Intentionalitätsweisen aber nun eine bestimmte menschliche personale Substanz mit welchen intentionalen Objekten zu welchen Zeiten erfüllt, geht aus dem Weltereignis hervor, gehört zu dessen Inhalt, ohne dass freilich die fragliche Substanz und jedenfalls die meisten ihrer intentionalen Objekte in einem unmittelbaren Sinn Teile des Weltereignisses wären (eine notwendige Bedingung dafür wäre ja, dass sie Ereignisse wären - was sie nicht sind). Die Substanz wird vom Weltereignis vielmehr innerlich impliziert, es verweist aus sich auf sie. ${ }^{27}$ Das kann es nur deshalb, weil die Substanz ein Manifestationsereignis, eine Geschichte hat (eine ontische, nicht notwendigerweise eine »aufgeschriebene Geschichte $\ll$ ), durch welches (welche) die Substanz im Weltereignis verankert ist (ihr Manifestationsereignis ist ja - im unmittelbaren Sinn - Teil des Weltereignisses). - Es ist hier nicht der Ort, näher über die genaue Zusammensetzung des Manifestationsereignisses einer menschlichen personalen Substanz zu sprechen; zentrale Teile ihres Manifestationsereignisses sind aber jedenfalls die Gesamtheit all ihrer Erlebnisse: ihr Bewusstseinsstrom, und die Gesamtheit all ihrer Wirkungen: ihr Wirken. Beide hängen, wie deutlich geworden sein dürfte, aufs Engste zusammen.

\section{Literaturverzeichnis}

Brentano, Franz 1973, Psychologie vom empirischen Standpunkt. Erster Band, hrsg. v. O. Kraus, Hamburg.

Carnap, Rudolf 1956, Meaning and Necessity, Chicago/London.

- 1962, Logical Foundations of Probability, Chicago/London.

Hume, David 1962, A Treatise of Human Nature. Book One, hrsg. v. D. G. C. Macnabb, Glasgow.

James, William 1950, The Principles of Psychology. Volume One, New York.

Leibniz, Gottfried Wilhelm 1985, Kleine Schriften zur Metaphysik, hrsg. v. H. H. Holz, Darmstadt.

Lewis, David 1986, On the Plurality of Worlds, Oxford.

Meixner, Uwe 1997, Ereignis und Substanz, Paderborn.

- 2002, »Change and Change-Ersatz«, in: A. Bottani u. a. (Hrsg.), Individuals, Essence and Identity, Dordrecht, 427-449.

- 2004a, Einfïhrung in die Ontologie, Darmstadt.

$2 \tau$ Wenn man sich freilich angewöhnt hat, im Weltereignis nur matter in motion zu sehen oder, alternativ, nur a flux of perceptions, dann ist einem diese Einsicht verwehrt. 
- 2004b, The Two Sides of Being, Paderborn.

- 2006, David Lewis, Paderborn.

- 2016, »Die fundamentale Zusammensetzung der Gegenstände und die Einfachheit Gottes. Eine axiomatisch-logische Rekonstruktion «, in: T. Marschler und T. Schärtl (Hrsg.), Eigenschaften Gottes. Ein Gespräch zwischen systematischer Theologie und analytischer Philosophie, Münster, 153-230.

Schnieder, Benjamin 2004, Substanzen und (ihre) Eigenschaften, Berlin/New York.

- 2005, »Substanz und Unabhängigkeit«, in: K. Trettin (Hrsg.), Substanz. Neue Überlegungen zu einer klassischen Kategorie des Seienden, Frankfurt a. M., 53-79.

Schütz, Ludwig 1958, Thomas-Lexikon, Stuttgart. [Faksimile-Neudruck der Ausgabe von 1895]

Tegtmeier, Erwin 1992, Grundzüge einer kategorialen Ontologie, Freiburg/München. Thomas von Aquin 1988, Summa Theologiae, Mailand. 